\title{
Retinal Ganglion Cell Layer Thinning within One Month of Presentation for Optic Neuritis
}

\author{
Mark J. Kupersmith, MD ${ }^{1}$, Mona K. Garvin, $\mathrm{PhD}^{2,3}$, Jui-Kai Wang, $\mathrm{MS}^{2}$, Mary Durbin, $\mathrm{PhD}^{4}$, \\ and Randy Kardon, MD, PhD $^{3}$ \\ ${ }^{1}$ New York Eye and Ear Infirmary, Mount Sinai Roosevelt Hospital, Icahn School of Medicine at \\ Mount Sinai, NY, NY \\ ${ }^{2}$ The Department of Electrical and Computer Engineering, The University of lowa, lowa City, IA \\ ${ }^{3}$ Department of Ophthalmology, lowa University School of Medicine and Center for Prevention \\ and Treatment of Visual Loss, Veterans Administration, lowa City, IA \\ ${ }^{4}$ Carl Zeiss-Meditec, Inc., Dublin, CA
}

\begin{abstract}
Background-Spectral domain optical coherence tomography (SD-OCT) reveals retina ganglion cell layer plus inner plexiform layer (GCL+IPL) and peripapillary nerve fiber layer (pRNFL) thinning in chronic optic nerve injury. At presentation, swelling of the pRNFL confounds evaluation of early axon loss.
\end{abstract}

Objective-We studied whether the GCL+IPL thins before the pRNFL, the trajectory of GCL +IPL loss and relationship to vision.

Methods-We prospectively evaluated 33 eyes (study) with new optic neuritis, using perimetry and SD-OCT with investigative 3-D layer segmentation and commercial 2-D segmentation to compute the GCL+IPL and pRNFL thickness.

Results-At presentation, GCL+IPL thickness $(82.4 \pm 8.8 \mu \mathrm{m})$ did not differ from unaffected fellow eyes $(81.2 \pm 6.7 \mu \mathrm{m})$, via the 3-D method, while the 2-D method failed in $9 \%$ of study eyes. At one-two months, there was thinning of pRNFL in 10\% and of GCL+IPL in $93 \%$ of study eyes. GCL+IPL reduction was greatest during the first two months. GCL+IPL thinning at one-two

Corresponding author: Mark J. Kupersmith, MD, mkuper@chpnet.org, Phone 212-636-3200, FAX 212-636-3195.

Author roles

Mark K. Kupersmith, MD - study concept and design, data collection, biostatistics and manuscript preparation, study supervision Mona K. Garvin, $\mathrm{PhD}$ - data analyses and manuscript preparation

Jui-Kai Wang, MS - data analyses and manuscript preparation

Mary Durbin, $\mathrm{PhD}$ - manuscript preparation, revision for intellectual content

Randy Kardon, MD, PhD - biostatistics and manuscript preparation, revision for intellectual content

Disclosures

Mark K. Kupersmith, MD - none

Mona K. Garvin, PhD - none

Jui Kai Wang, MS - none

Mary Durbin, PhD - employee of Zeiss-Meditec, Inc.

Randy Kardon, MD, PhD - none 
months correlated with GCL+IPL thinning at 6 months $(\mathrm{r}=0.84, \mathrm{p}=0.01)$ and presentation visual acuity $(\mathrm{r}-0.48, \mathrm{p}=0.006)$ and perimetric mean deviation $(\mathrm{r}=0.52, \mathrm{p}=0.003)$.

Conclusion-GGL+IPL is an early biomarker of structural injury in optic neuritis as thinning develops within one-two months of onset, prior to pRNFL thinning.

\section{Keywords}

retina ganglion cell layer; periapillary retina nerve fiber layer; RNFL; OCT; optic neuritis

\section{Introduction}

Acute optic neuritis $(\mathrm{ON})$ causes vision loss at presentation that recovers in the majority of affected eyes. Optic nerve head swelling can last more than a month, making it difficult for optical coherence tomography (OCT) to demonstrate if and when permanent structural injury to the optic nerve and loss in the peripapillary retinal nerve fiber layer (pRNFL) have occurred. ${ }^{1}$ Despite these limitations, optic neuritis can serve as in vivo injury model of an acute demyelinating lesion to study and evaluate the effectiveness of potential neuroprotective agents on quantitative functional and structural outcome measures. ${ }^{2,3}$

OCT shows pRNFL thinning correlates with visual field loss in other optic neuropathies, glaucoma and ischemic optic neuropathy. ${ }^{4-12}$ In glaucoma, thinning of the retinal ganglion cell layer (GCL) plus inner plexiform layer (IPL) occurs with the thinning of pRNFL ${ }^{13,14}$ and reflects progressive injury but both measures do not always change in parallel. ${ }^{15}$ Determination of the thickness of the GCL alone or in combination with the IPL as is typically performed, also reveals thinning with long standing multiple sclerosis ${ }^{16,17}$ and following non-arteritic anterior ischemic optic neuropathy. ${ }^{18}$ Given that approximately $50 \%$ of retinal ganglion cells are located in the human macula region ${ }^{19}$ and optic neuritis tends to affect some portion of the central 10 degrees of visual field, GCL+IPL measurement might detect early structural loss.

We postulated the GCL+IPL measurement at presentation would not be confounded by optic nerve head, pRNFL or thickening of retinal layers not including the GCL, and could be a reliable baseline structural biomarker that could be followed longitudinally.

\section{Methods}

We prospectively evaluated eyes (study eyes) with new onset optic neuritis ( $\mathrm{n}=33$ ), within 15 days of patient-reported vision loss, at one-two months after vision loss (or approximately one month from presentation), at three months and at six months or longer from 2009-2013. Each subject had clinical evaluation and perimetry performed using the Humphrey Field Analyzer (Zeiss-Meditec, Inc., Dublin, CA) with SITA 24-2 standard perimeter strategy using size III (expressed as perimetric mean deviation, PMD, in decibels, $\mathrm{dB}$ ), and spectral domain (SD) OCT of the optic disc and macula regions at each visit (details below). Visual acuity was reported as LogMar values. Gadolinium-enhanced magnetic resonance image (1.5T) of the brain and orbits was performed, which included fat suppression and short tau inverted recovery (STIR) sequences for the orbits (within several days of presentation) in 32 
patients (one had prior MRI). The hyperintense signal on the STIR images and/or the abnormal enhancement on T1 images were used to determine the length of the optic nerve lesion on MRI. Fellow eyes were considered normal if there was no history of optic neuritis or abnormal signal in the optic nerve on MRI, visual acuity 20/25 or better, normal 24-2 visual field, and the average pRNFL thickness $\geq 5^{\text {th }}$ percentile of the Zeiss-Meditec (ZM) controls. This research was conducted with New York Eye and Ear Infirmary IRB approval and adhered to the tenets of the Declaration of Helsinki.

Following pupillary dilation, we used the Cirrus 4000 SD-OCT (Zeiss-Meditec, Dublin, CA) to scan a $6 \times 6 \mathrm{~mm}$ area, to capture volume data consisting of 200 A-scans from 200 linear bscans for the optic nerve region, and 128-b scans, each with 512 pixels of horizontal width and 1024 pixels of vertical height for the macula region. At least two scans were obtained for each region and only images centered on the optic disc or macula with signal strength scores 6 or greater were analyzed. The average peripapillary pRNFL thickness (microns, $\mu \mathrm{m})$ was calculated from values at 256 points in the peripapillary circumference using the Zeiss-Meditec algorithm and exporting process. The average GCL+IPL thicknesses $(\mu \mathrm{m})$ was derived from macula scans using, two algorithms: a three dimensional (3D) segmentation methodology developed at the University of Iowa ${ }^{21}$ (3-D method) and the Zeiss-Meditec (ZM) proprietary methodology (2-D method), utilizing Cirrus software 6.0.2.

For 3-D method, the GCL+IPL complex thickness (excluding the local RNFL) was calculated from eleven intra-retinal surfaces of each macula-centered volumetric scan that were first segmented using a previously published graph-theoretic approach. ${ }^{20} \mathrm{~A}$ minimum of four surfaces including interfaces between layers were retained to enable computation of the fovea center and the combined GCL+IPL thickness. The mean GCL+IPL thickness was computed within an elliptical annulus (with a vertical inner and outer radius of $0.5 \mathrm{~mm}$ and $2.0 \mathrm{~mm}$, respectively; and horizontal inner and outer radius of $0.6 \mathrm{~mm}$ and $2.4 \mathrm{~mm}$, respectively) centered at the fovea.

The 2-D method utilized a two dimensional proprietary algorithm which finds the distance between the outer boundary of the RNFL and the outer boundary of the IPL to report the combined thickness of the GCL+IPL. ${ }^{11}$ The average thickness of the GCL+IPL is measured in an elliptical annulus of the same dimension of the 3D-method described above. The raw image data are initially segmented identifying the inner limiting membrane (ILM) and retinal pigment epithelium to create a region of interest within which lie the intra-retinal layers. The algorithm continues this hierarchical approach, segmenting first the outer boundary of the outer plexiform layer, followed by the outer boundary of the IPL, and last the outer boundary of the RNFL.

We measured the average pRNFL thickness and the macula region GCL+IPL thickness in the normal fellow eyes of study subjects using the 3-D method. These eyes had a mean GCL + IPL $81.2 \pm 6.7 \mu \mathrm{m}$ and the $5^{\text {th }}$ percentile lower limit was $70.8 \mu \mathrm{m}$. We also utilized the ZM control data base (which included 149 women and 133 men with a mean age of $46.6 \pm 16.9$ years) and calculated the age related $5^{\text {th }}$ and $95^{\text {th }}$ percentile of the pRNFL thickness values (83.0 $\mu \mathrm{m}$ and $113.5 \mu \mathrm{m}$, respectively) and of the GCL+IPL thickness (for 3-D method) values ( $71.8 \mu \mathrm{m}$ and $93.4 \mu \mathrm{m}$, respectively) for the 3D-method. We calculated the variability 
for the 3-D method GCL+IPL measurement in the normal fellow eyes by retesting on at least three visits over several months (Figure 1). These data were used to determine the $5^{\text {th }}$ and $95^{\text {th }}$ percentile normal limits. These normal fellow eyes had a mean change between visits of $0.03 \pm 1.2 \mu \mathrm{m}$ and the $95^{\text {th }}$ percentile for change was $1.9 \mu \mathrm{m}$. Reduction in the study eye GCL+IPL measurement over time greater than $1.9 \mu \mathrm{m}$ was therefore utilized to determine thinning of the GCL+IPL.

We report the average pRNFL (2-D method only) and GCL+IPL (both methods) thickness measurements over six months. We compared the average GCL+IPL thickness measurements for the 3-D and 2-D methods. We report the amount of GCL+IPL thickness reduction compared with baseline for 3-D method using t-tests. Pearson correlations were used for comparing visual acuity, PMD and MRI optic nerve lesion with the 3D method derived GCL+IPL reduction at one-two months and six months.

\section{Results}

The patients, 11 men and 22 women, with a mean age $37 \pm 12$ years, were evaluated on average at $7.3 \pm 3.4$ days from the reported onset of vision loss. The visual acuity, visual field loss and recovery were typical of optic neuritis (Table 1). No patient had diagnosed multiple sclerosis prior to presentation; seven had prior optic neuritis in the fellow eye, three were diagnosed as having MS at presentation, and 10 had a clinically isolated syndrome with white matter hyperintense lesions in the T2 and FLAIR sequences of the brain MRI. Abnormal signal and/or enhancement was seen in all study optic nerves that had fat suppressed gadolinium T1 weighted and short tau inversion recovery studies of the orbits. The average pRNFL thickness measurement at presentation was $116 \pm 7.8 \mu \mathrm{m}$. pRNFL thickening at presentation (Table 1) occurred in 10 of 33 study eyes (defined as pRNFL thickness $>95^{\text {th }}$ percentile for 32 year old ZM controls) and $46 \%$ of study eyes had pRNFL values $10 \%$ or greater than the fellow normal eye. At presentation, no study eye had pRNFL thinning (defined as pRNFL thickness $<5^{\text {th }}$ percentile for 32 year old ZM controls).

The 3-D and 2-D methods gave similar results for GCL+IPL measurement in 26 unaffected fellow normal eyes of the study subjects. For the same dates, the values from both methods showed a strong correlation $(r=0.96, p=0.001)$.

In three study eyes, 2-D method values $(50 \pm 2.0 \mu \mathrm{m})$ for GCL+IPL were markedly less than 3 -D method $(77.8 \pm 3.1 \mu \mathrm{m} ; \mathrm{p}=0.001)$ values at presentation. All three eyes had 2-D method GCL+IPL values $\leq 20 \mu \mathrm{m}$ than the values derived by 3 -D method. The correlation coefficient for the baseline GCL+IPL thickness in all 33 eyes between 3-D and 2-D measures was 0.51 ( $\mathrm{p}=0.002$, Figure 2). Excluding the three eyes with presumed artifactual GCL+IPL reduction by 2-D method at presentation, the GCL+IPL values derived from both methods showed a strong correlation $(r=0.87, \mathrm{p}=0.01)$.

At presentation (acute stage), the 3-D method showed no significant thinning or thickening of GCL+IPL thickness in study eyes compared with normal fellow eyes. The mean difference in GCL+IPL thickness between the study and unaffected eyes was $0.75 \pm 5.1 \mu \mathrm{m}$. 
No study eyes had GCL+IPL thickness less than the $5^{\text {th }}$ percentile of the normal eyes from $\mathrm{ZM}$ or normal fellow eyes $(73.1 \mu \mathrm{m})$ using the 3-D method.

We evaluated 10 study eyes in patients who returned earlier than one month for examination (within 21 days of presentation, mean $15.6 \pm 5.0$ days) or at a mean time after vision loss of $21.8 \pm 6.0$ days. The GCL+IPL thickness was reduced, on average $8.4 \pm 5.4 \mu \mathrm{m}$ (range 15.9 $\mu \mathrm{m}$ ), from presentation values in nine eyes (Figure 3). Four eyes had GCL+IPL thickness below the normal $5^{\text {th }}$ percentile while none had RNFL values below the normal $5^{\text {th }}$ percentile.

At the one-two months exam (mean $30 \pm 12$ days from presentation), the average pRNFL thickness measurements for 30 study eyes was $99.1 \mu \mathrm{m}$ (Figure 4 and Table 1). The average pRNFL became thinner in 16 study eyes at one-two months due to reduction in optic nerve edema from presentation (mean pRNFL reduction $=22 \pm 44 \mu \mathrm{m}$ ). Only three eyes $(10 \%)$ had pRNFL thinned less the $5^{\text {th }}$ percentile of controls ( $83 \mu \mathrm{m}$ for 32 year olds). For 28 of 30 (93\%) study eyes, the GCL+IPL 3D method measurement at one-two months was less than baseline (reduced by $>1.9 \mu \mathrm{m}$ ), an average of $9.9 \pm 6.1 \mu \mathrm{m}(\mathrm{p}=0.001$; Figure 5$)$. The GCL + IPL thickness was less than the control $5^{\text {th }}$ percentile $(71.8 \mu \mathrm{m})$ in $50 \%$ of study eyes. The GCL+IPL thinning at one-two months accounted for an average of $77 \% \pm 20 \%$ of the total thinning that occurred over the six months.

At one-two months, the GCL+IPL thickness values for all study eyes were similar for the 3D and 2D methods $(r=0.85, p=0.001$; Table 1$)$. The three eyes with low baseline GCL+IPL thickness by the 2D-method were at least $12 \mu \mathrm{m}$ thicker at one-two months. The 3D-method derived GCL+IPL thinning at one-two months showed no correlation with the PMD or visual acuity at that time point. The amount of GCL+IPL thinning at one-two month did not correlate with duration of vision loss at presentation nor with the vision at one-two months-, but it did show moderate correlation with the presentation visual acuity $(\mathrm{r}=-0.48, \mathrm{p}=0.006)$ and PMD $(r=0.52, \mathrm{p}=-.003)$ and with the six month PMD $(\mathrm{r}=0.38, \mathrm{p}=0.04)$.

Compared to baseline, the 3D-method GCL+IPL showed total thinning at three months (mean $118 \pm 37$ days from presentation) an average of $11.4 \pm 8.4 \mu \mathrm{m}(\mathrm{p}=0.01)$ and $13.5 \pm 8.9$ $\mu \mathrm{m}(\mathrm{p}=0.01)$ at six months (mean $291 \pm 126$ days from presentation; Figure 5 and Table 1). The 2-D method GCL+IPL thickness values were also reduced (Table 1) and the GCL+IPL thickness measurements by the 3D and 2D methods were strongly correlated at three $(\mathrm{r}=0.99, \mathrm{p}=0.01)$ and six months $(\mathrm{r}=0.98, \mathrm{p}=0.01)$. At six months, the average $\mathrm{pRNFL}$ thickness for study eyes $(84.1 \pm 11.2 \mu \mathrm{m})$ was thinned compared to the normal fellow eyes $(93.1 \pm 9.0 \mu \mathrm{m}, \mathrm{p}=0.01)$; and 17 eyes had pRNFL thickness less than the control eye $5^{\text {th }}$ percentile of $83 \mu \mathrm{m}$ (Figure 4). At six months, all study eyes had 3D-method GCL+IPL thinning greater than $-1.9 \mu \mathrm{m}$ compared to baseline presentation and $56 \%$ were thinner than the $5^{\text {th }}$ percentile for normal eyes $(71.8 \mu \mathrm{m})$. At six months, the amount of pRNFL and GCL + IPL thinning (relative to the normal fellow eye) were moderately correlated $(r=0.48, p=$ $0.02)$. The three and six month GCL+IPL thickness were strongly correlated $(r=1.00, p=$ 0.01 ) and the mean loss was only $0.59 \mu \mathrm{m}+0.96$ between the three and sixth month exams. 
GCL+IPL thinning at one-two months and six months were strongly correlated $(\mathrm{r}=0.84$, $\mathrm{p}=0.01$ ). Neither the GCL+IPL thickness value or amount of thinning or the pRNFL thickness value at six months correlated with the visual acuity or PMD at six months. However, there was moderate correlation between the six month GCL+IPL reduction and the presenting visual acuity $(\mathrm{r}=0.50, \mathrm{p}=0.003)$ and $\mathrm{PMD}(\mathrm{r}=0.46, \mathrm{p}=0.008)$.

\section{MRI correlations with $p R N F L$ and GCL+IPL (Figure 6)}

Study eyes showed a moderate correlation for GCL+IPL thinning at one-two months with the length of the optic nerve lesion $(r=0.45, p=0.014)$ on MRI short tau inversion recovery or gadolinium enhanced T1 sequences. There was no correlation for GCL+IPL thinning at one-two months and the proximity of the lesion to the globe $(r=0.24, p=0.19)$ by MRI.

\section{Discussion}

This study shows the importance of measuring the GCL+IPL thickness as a structural biomarker for evaluating the effects of optic neuritis. GCL+IPL thinning, which represents permanent shrinkage or loss of retinal ganglion cells, occurs at one month and possibly earlier. This confirms the results from a study recently reported on acute optic neuritis. ${ }^{21}$ In contrast to the early GCL+IPL loss, significant pRNFL loss cannot be detected until three months due to acute thickening, which prevents detection of early axon loss. GCL+IPL thinning worsens over six months but the trajectory of loss is greatest during the first month. The 3-D segmentation algorithm appears to be superior to the 2D commercial algorithm for accurately measuring GCL+IPL thickness at presentation when pRNFL swelling is prominent. In a small group of patients, who had evaluations earlier than the one-two month time point, GCL+IPL thinning developed in $90 \%$ of eyes within two to three weeks after presentation. Additional cases need to be evaluated weekly for the first month after vision loss in order to verify this finding and determine the earliest time when GCL+IPL loss occurs.

Even though the trajectory for thickness reduction appears similar for GCL+IPL and pRNFL, it is important to note that pRNFL thinning below the $5^{\text {th }}$ percentile of normal eyes occurs later than GCL+IPL thinning. At one to two months after optic neuritis onset, pRNFL thinning less than normal occurred in $10 \%$ of eyes, while GCL+IPL thinning occurred in $50 \%$ of eyes. By three months, thinning of the pRNFL and GCL+IPL occurred in 50\% and $57 \%$ of eyes, respectively. Most GCL+IPL thinning occurs during the first two months, possibly due to early changes in the morphology of the retinal ganglion cell bodies and dendrites. ${ }^{22-24}$

The GCL+IPL thickness determined with 3D-segmentation was not thinned at presentation. However, the 2-D method for determining GCL+IPL thickness failed in 9\% of study eyes, presumably due to processes that distort normal retinal layer architecture and adversely affect the proprietary segmentation algorithm. The 2-D method showed a similar apparent failure when the optic nerve head is markedly swollen as in papilledema. ${ }^{25}$ At the one-two month follow up evaluation the apparent GCL+IPL thinning found using the 2-D method normalized, suggesting the baseline results using the 2-D method were flawed. In contrast, 
the 3-D method appeared to be relatively unaffected by the same pathological processes at presentation. The 2-D method algorithm performs two dimensional segmentation and assumes a quantitative relationship between the internal limiting membrane and the other layers of the retina. The 2-D method appears more susceptible to failure with any process, such as edema due to swelling of the peripapillary pRNFL and adjacent retina, which disrupts the regular retinal layer position, shape and boundaries. In contrast, the 3-D method algorithm incorporates 3D contextual information into the optimization process which helps to reduce failures due to local distortions in retinal layers. It is clinically important to carefully evaluate algorithm performance in OCT scans, since apparent failures may lead to false interpretations and may adversely influence clinical decisions.

OCT demonstration of pRNFL and GCL+IPL thinning is important in determining irreversible neuronal loss. Retinal ganglion cell loss has been shown on histopathology in glaucoma ${ }^{24,26}$ and in eyes of patients with multiple sclerosis, even without a history of optic neuritis. ${ }^{27}$ To date, one month has been considered the earliest time point for demonstrating vision or pRNFL features that predict permanent injury in optic neuritis. ${ }^{2,28,29}$ Our results suggest that permanent structural loss or atrophy of RGCs commonly occurs by one-two months, and even earlier in some cases, with acute optic neuritis. We cannot directly compare our finding with other reports on optic neuritis. A prior report, by Syc et al. ${ }^{30}$, described GCL+IPL thinning with acute optic neuritis evaluated within four weeks of vision loss but was possibly complicated by the use of a commercial algorithm similar to the 2-D method which fails when optic nerve head swelling is present. Also, prior optic nerve injury might have been present since $45 \%$ of their patients had MS for five years. ${ }^{30} \mathrm{GCL}+\mathrm{IPL}$ thinning of approximately $10 \mu \mathrm{m}$ was seen at three months, similar to what we found at onetwo months, but earlier results were not reported.

MRI optic nerve lesion length, but not lesion proximity to the globe, correlated with GCL +IPL thinning at one to two months. Prior studies showed longer MRI lesions were associated with more severe vision loss at presentation, ${ }^{31,32}$ suggesting a more profound injury. However, neither the length of lesion or GCL+IPL thinning at one-two months correlated with vision outcome. ${ }^{31}$ We had anticipated greater GCL+IPL thinning would occur earlier when the lesion was closer to the eye and the RGC's, but this was not shown. One possible explanation is that GCL+IPL thinning is not just a manifestation of retrograde degeneration occurring from the site of the inflammation. Additional pathological process such as intra-retinal release of toxic excitatory neurotransmitters could be contributory.

pRNFL and GCL atrophy or loss correlates particularly when there is chronic disease or significant persistent vision loss in glaucoma, ischemic optic neuropathy, optic neuritis and MS. ${ }^{13,14,16-18,26}$ In our study, vision loss was not permanent as is the case in most optic neuritis. The lack of correlation between the visual field or visual acuity and the amount of GCL+IPL loss or preservation at six months, when the episode is considered to be concluded, suggests that a more sensitive method of vision performance such as low contrast acuity might be needed particularly when investigating eyes with optic neuritis that typically recover ${ }^{33,34}$. Additionally, overlap in receptor fields and redundancy of neural networks in the macula that support normal vision function may not require the full complement of RGCs. 
GCL+IPL thickness measurement appears to be a better biomarker of structural injury than pRNFL thickness with acute optic nerve injury due to optic neuritis. The 3D-segmentation algorithm can accurately measure the GCL+IPL at baseline and the change over time.

\section{Acknowledgments}

This work was supported, in part, by 3U10EY017281-04AS1, NEI subcontract R009040554, NEI R01 EY018853, R01 EY023279, the Department of Veterans Affairs Rehabilitation Research and Development Division (Iowa City Center for the Prevention and Treatment of Visual Loss and Career Development Award 1IK2RX000728 for Dr. Garvin), and Research to Prevent Blindness (New York, New York)

\section{References}

1. Kupersmith M, Mandel G, Anderson S, Meltzer D, Kardon R. Baseline and one month changes in the peripapillary retinal nerve fiber layer in acute optic neuritis: Relation to baseline vision and MRI. J Neurol Sci. 2011; 308:117-123. [PubMed: 21764408]

2. Kupersmith M, Gal R, Beck R, Xing D, Miller N, The Optic Neuritis Study Group. Visual function at baseline and one month in acute demyelinating optic neuritis predicts visual outcome. Neurology. 2007; 69:508-514. [PubMed: 17679669]

3. Henderson A, Altman D, Trip A, Kallis C, Jones S, Schlottman P, Garway-Heath D, Plant G, Miller D. A serial study of retinal changes following optic neuritis with sample size estimates for acute protection trials. Brain. 2010; 33:292-2602.

4. Raza A, Jungsuk C, de Moraes C, Wang M, Zhang Z, Kardon R, Liebmann J, Ritch R, Hood D. Retinal ganglion cell layer thickness and local visual field sensitivity in glaucoma. Arch Ophthalmol. 2011; 129:1529-1536. [PubMed: 22159673]

5. Takagi S, Kita Y, Fumihiko Y, Tomita G. Macular retinal ganglion cell complex damage in the apparently normal visual field glaucomatous eyes with hemifield defects. J Glaucoma. 2012; 21:318-325. [PubMed: 21423034]

6. Costello F, Coupland S, Hodge W, Lorello G, Koroluk J, Pan I, Freedman M, Zackon D, Kardon R. Quantifying axonal loss after optic neuritis with ocular coherence tomography. Ann Neurol. 2006; 59:963-969. [PubMed: 16718705]

7. Trip A, Schlottmann P, Jones S, Altmann D, Garway-Heath D, Thompson A, Plant G, Miller D. Retinal nerve fiber layer axonal loss and visual dysfunction in optic neuritis. Ann Neurol. 2005; 58:383-391. [PubMed: 16075460]

8. Danish-Meyer H, Carroll S, Ku J, Hsiang J, Gaskin B, Gamble G, Savino P. Correlation of retinal nerve fiber layer measured by scanning laser polarimeter to visual field in ischemic optic neuropathy. Arch Ophthal. 2006; 124:172-1726.

9. Contrearas I, Noval S, Rebolleda G, Munoz-Negrete F. Follow-up of nonarteritic anterior ischemic optic neuropathy with optical coherence tomography. Ophthalmology. 2007; 114:2338-2344. [PubMed: 17719640]

10. Hood D, Anderson S, Rouleau J, Wenick A, Grover L, Behrens M, Odel J, Lee A, Kardon R. Retinal nerve fiber structure versus visual field function in patients with ischemic optic neuropathy. Ophthalmology. 2008; 115:904-910. [PubMed: 17870170]

11. DeLeon-Ortega J, Carrol K, Arthur S, Girkin C. Correlations between retinal nerve fiber layer and visual field in eyes with nonarteritic anterior ischemic optic neuropathy. Am J Ophthalmol. 2007; 143:288-294. [PubMed: 17157797]

12. Bellusci C, Savini G, Carbonelli M, Carelli V, Sadaun A, Barboni P. Retinal nerve fiber layer thickness in nonarteritic anterior ischemic optic neuropathy: OCT characterization of the acute and resolving phases. Graefs Arch Clin Exp Ophthalmol. 2008; 246:641-647.

13. Mwanza JC, Durbin M, Budenz D, Sayyad F, Chang R, Neelalantam A, Godfrey D, Carter R, Crandall A. Glaucoma diagnostic accuracy of ganglion cell-inner plexiform layer thickness: comparison with nerve fiber layer and optic nerve head. Ophthalmology. 2012; 119:1151-1158. [PubMed: 22365056] 
14. Koh V, Tham YC, Cheung C, Wong WL, Baskaran M, Saw SM, Wong T, Aung T. Determinants of ganglion cell-ner plexiform layer thickness measured by high-definition optical coherence tomography. Invest Ophthalmol Vis Sci. 2012; 53:5853-5859. [PubMed: 22836772]

15. Na JH, Kook M, Lee Y, Baek S. Structure-function relationship of the macular visual field and the ganglion cell complex thickness in glaucoma. Invest Ophthalmol Vis Sci. 2012; 53:5044-5051. [PubMed: 22700706]

16. Fisher JB, Jacobs DA, Markowitz CE, Galetta SL, Volpe NJ, Nano-Schiavi ML, et al. Relation of visual function to retinal nerve fiber. Brain. 2012; 135:521-533. [PubMed: 22006982]

17. Walter S, Ishikawa H, Galetta K, Sakai R, Feller D, Henderson S, Wilson J, Maguire M, Galetta S, Frohman E, Calabresi P, Schuman J, Balcer L. Ganglion cell loss in relation to visual disability in multiple sclerosis. Ophthalmology. 2012; 119:1250-1257. [PubMed: 22365058]

18. Aggarwal D, Tan O, Huang D, Sadun A. Patterns of ganglion cell complex and nerve fiber layer loss in nonarteritic ischemic optic neuropathy by fourier-domain optical coherence tomography. Invest Ophthalmol Vis Sci. 2012; 53:4539-4545. [PubMed: 22678499]

19. Curcio C, Allen K. Topography of ganglion cells in human retina. J Comp Neurol. 1990; 300:5-25. [PubMed: 2229487]

20. Garvin MK, Abràmoff MD, Kardon R, Russell SR, Wu X, Sonka M. Intraretinal layer segmentation of macular optical coherence tomography images using optimal 3-D graph search. IEEE Transactions on Medical Imaging. 2008; 27:1495-1505. [PubMed: 18815101]

21. Gabilondo I, Martinez-Lapiscina E, Fraga-Pumar E, Ortiz-Perez S, Torres-Torres R, Andorra M, Llufriu S, Zubizarreta I, Saiz A, Sanchez-Dalmau B, Villoslada P. Dynamics of retinal injury after acute optic neuritis. Ann Neurol. 2015; 77:517-528. [PubMed: 25559267]

22. Kalesnykas G, Oglesby E, Zack D, Cone F, Steinhart M, Tian J, Pease M, Quigley H. Retinal ganglion cell morphology after optic nerve crush and experimental glaucoma. Invest Ophthalmol Vis Sci. 2012; 53:3847-57. [PubMed: 22589442]

23. Almasieh M, Wilson A, Morquette B, Cueva Vargas J, Di Polo A. The molecular basis of retinal ganglion cell death in glaucoma. Prog Retin Eye Res. 2012; 31:152-81. [PubMed: 22155051]

24. Feng L, Zhao Y, Yoshida M, Chen H, Yang J, Kim T, Cang J, Troy J, Liu X. Sustained ocular hypertension induces dendritic degeneration of mouse retinal ganglion cells that depends on cell type and location. Invest Ophthalmol Vis Sci. 2013; 54(2):1106-17. [PubMed: 23322576]

25. OCT Sub-Study Committee for the NORDIC Idiopathic Intracranial Hypertension Study Group. Baseline OCT Measurements in the Idiopathic Intracranial Hypertension Treatment Trial: Part I. Quality Control, Comparisons and Variability. Inv Ophthal Vis Sci. 2014; 55:8173-8179.

26. Lei Y, Garrahan N, Hermann B, Becker DL, Hernandez MR, Boulton ME, et al. Quantification of retinal transneuronal degeneration in human glaucoma: a novel multiphoton-DAPI approach. Invest Ophthalmol Vis Sci. 2008; 49:1940-5. [PubMed: 18436826]

27. Green A, McQuaid S, Hauser S, Allen I, Lyness R. Ocular pathology in multiple sclerosis: retinal atrophy and inflammation irrespective of disease duration. Brain. 2010; 133:1591-1601. [PubMed: 20410146]

28. Kupersmith M, Anderson S, Kardon R. Predictive value of 1 month retinal nerve fiber layer thinning for deficits at 6 months after acute optic neuritis. Multiple Sclerosis J. 2013; 19:17431748 .

29. Kupersmith M, Kardon R, Durbin M, Horne M, Schulman J. Scanning laser polarimetry reveals status of axon integrity in areas of optical coherence tomography revealed thickened retinal nerve fiber layer (RNFL) with optic nerve head swelling. Invest Ophthalmol Vis Sci. 2012; 53:19621970. [PubMed: 22410562]

30. Syc S, Saidha S, Newsome S, Ratchford J, Levy M, Ford E, Crainiceanu C, Durbin M, Oakley J, Meyer S, Frohman E, Calabresi P. Optical coherence tomography segmentation reveals ganglion cell layer pathology after optic neuritis. Brain. 2012; 135:521-533. [PubMed: 22006982]

31. Kupersmith M, Alban T, Zeiffer B, Lefton D. Contrast-enhanced MRI in acute optic neuritis: relationship to visual performance. Brain. 2002; 125:812-82. [PubMed: 11912114]

32. Miller D, Newton M, van der Poel J, du Boulay E, Halliday A, Kendall B, et al. Magnetic resonance imaging of the optic nerve in optic neuritis. Neurology. 1988; 38:175-179. [PubMed: 3340276] 
33. Beck RW, Cleary PA, Anderson MM, Kupersmith MJ, et al. A randomized, controlled trial of corticosteroids in the treatment of acute optic neuritis. N Engl J Med. 1992; 326:581-588. [PubMed: 1734247]

34. Sakai R, Feller D, Galetta K, Galetta S, Balcer L. Vision in multiple sclerosis: the story, structure, function correlations, and models for neuroprotection. J Neuro-Ophthalmol. 2011; 31:362-737. 


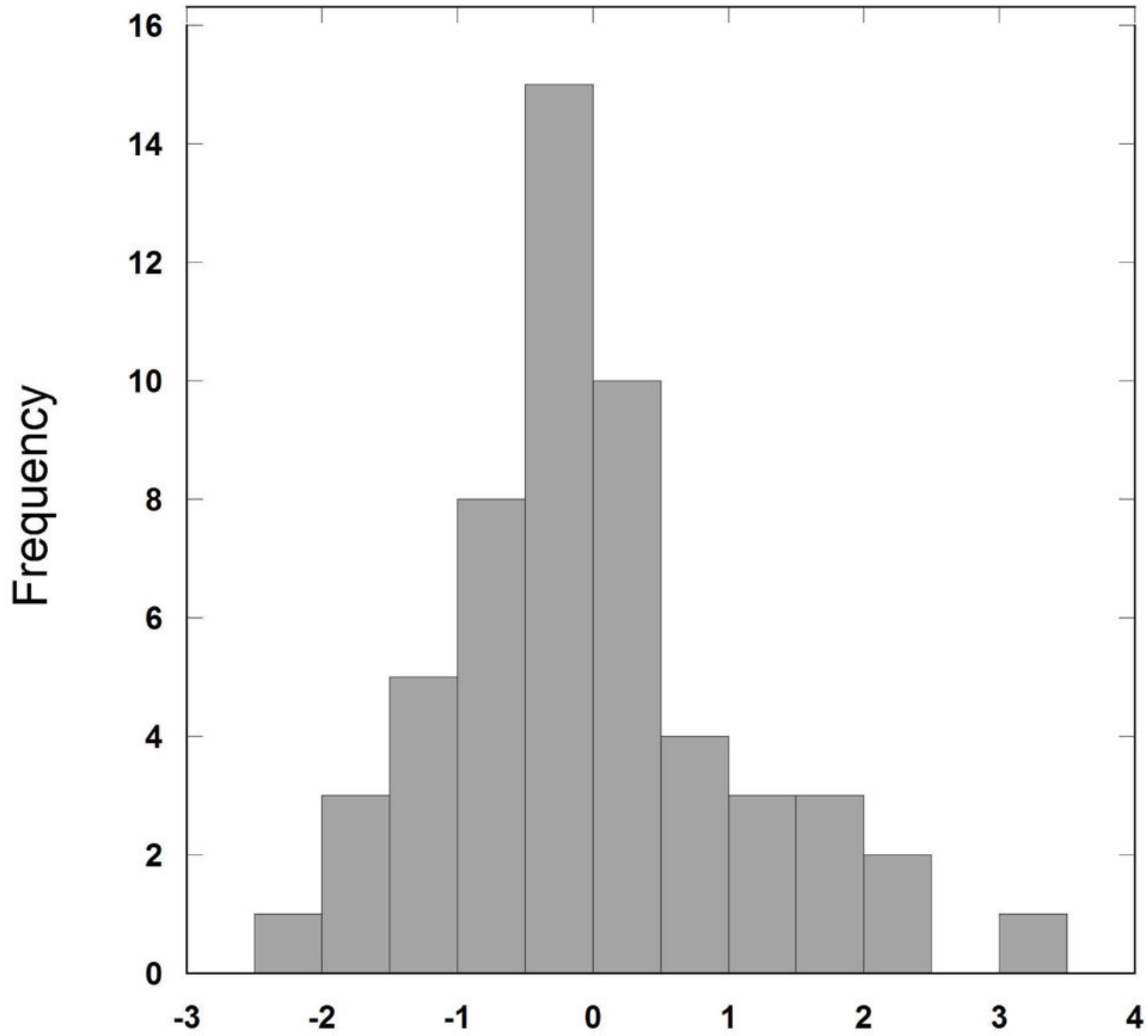

\section{GCL+IPL Thickness Reduction After Presentation (microns)}

Figure 1.

Frequency histogram of the change in 3-D method derived GCL+IPL thickness in microns in unaffected fellow eyes from the presentation thickness across two to three additional study visits. 


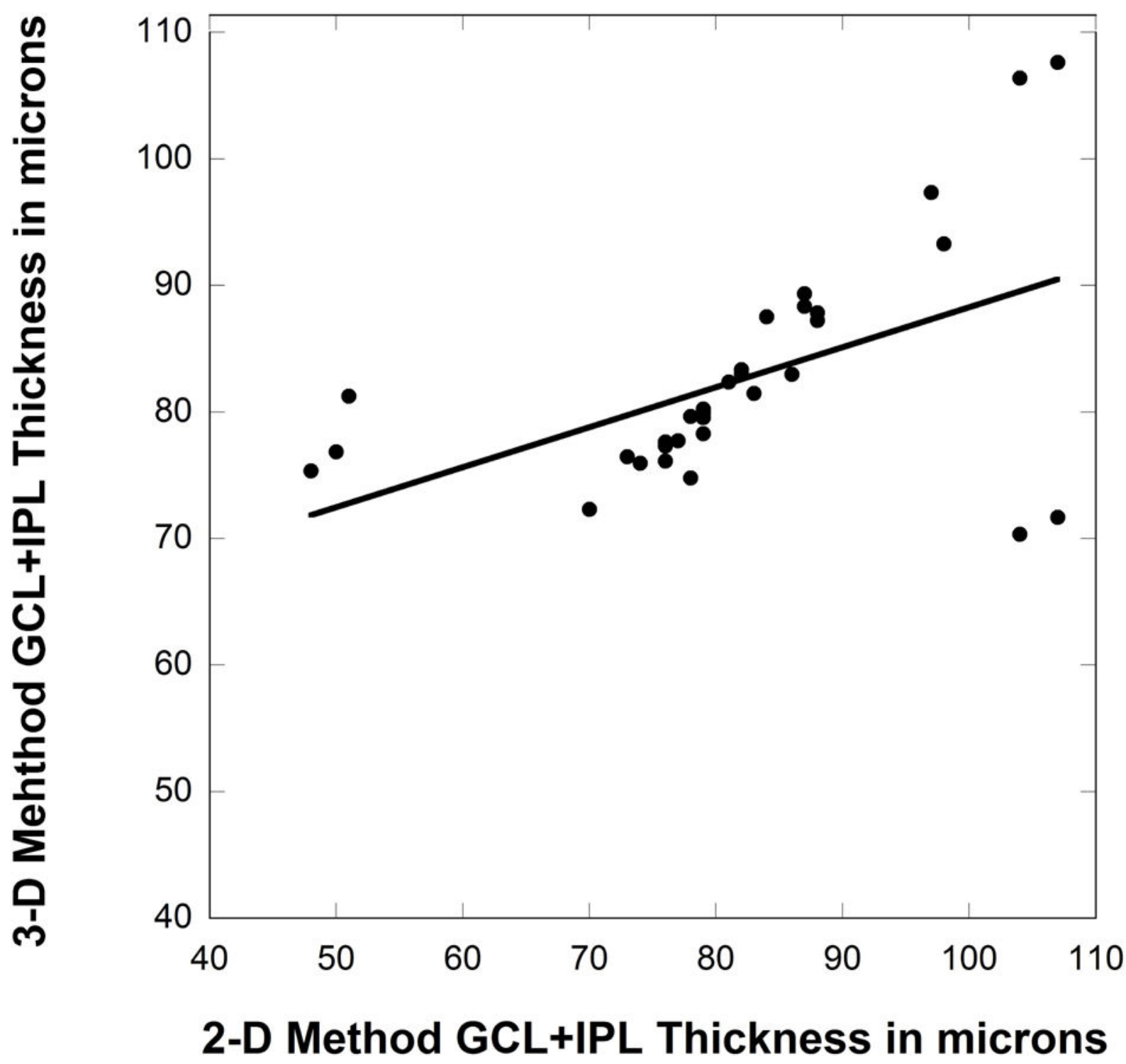

Figure 2.

Presentation GCL+IPL thickness derived by 3-D method and 2-D method for study optic neuritis eyes. The linear fit line formula: $y=56.7+0.316 \times R ; R=0.51$. 


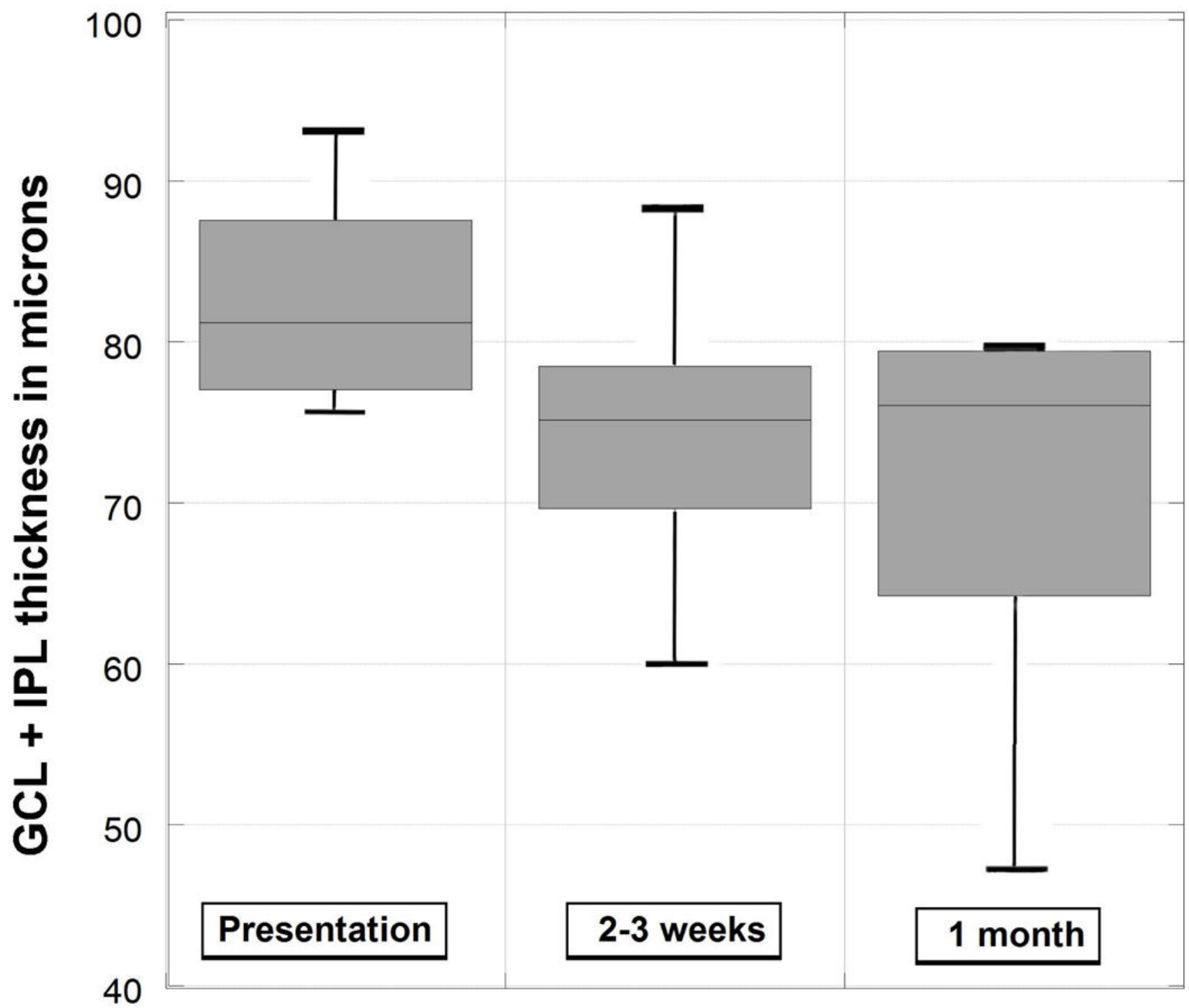

Figure 3.

Box plots of 3-D method derived GCL+IPL thinning for 10 study eyes evaluated within two to three weeks of acute presentation. 


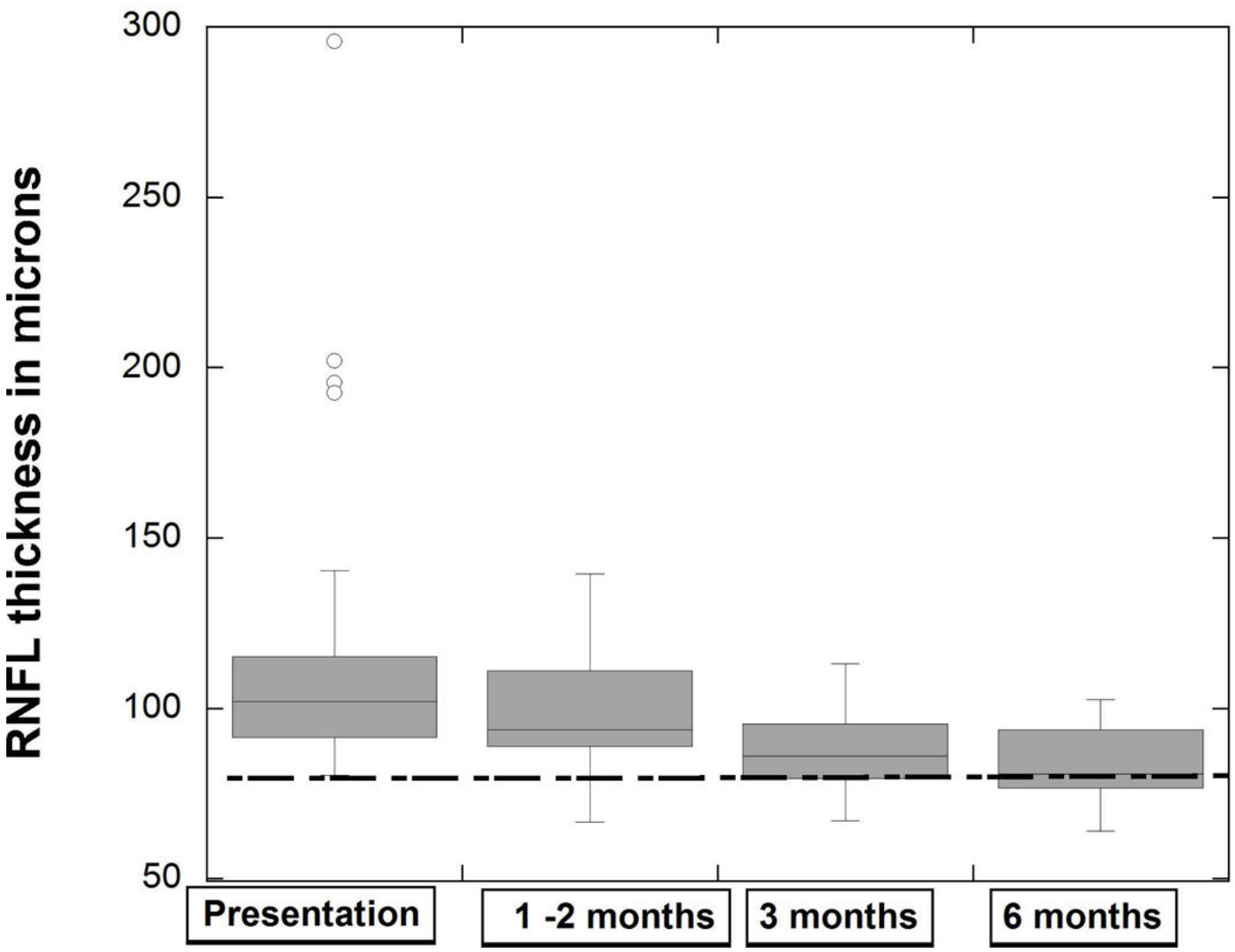

Figure 4.

Box plots of pRNFL thickness over six months for study eyes. The dashed line is the fifth percentile for control eyes. 


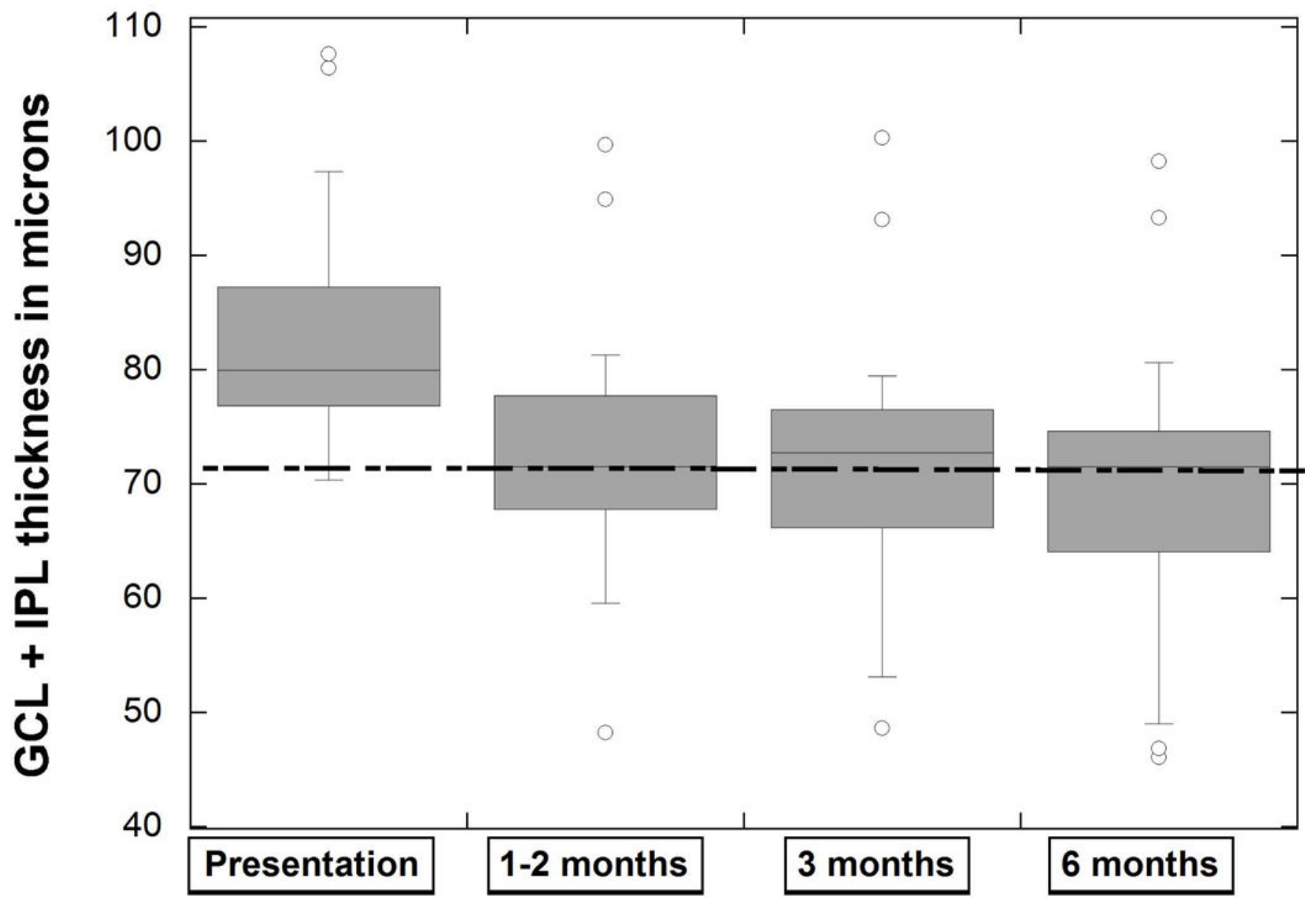

Figure 5.

Box plots of 3-D method derived GCL+IPL thickness over six months for study eyes. The dashed line is the fifth percentile for control eyes. 

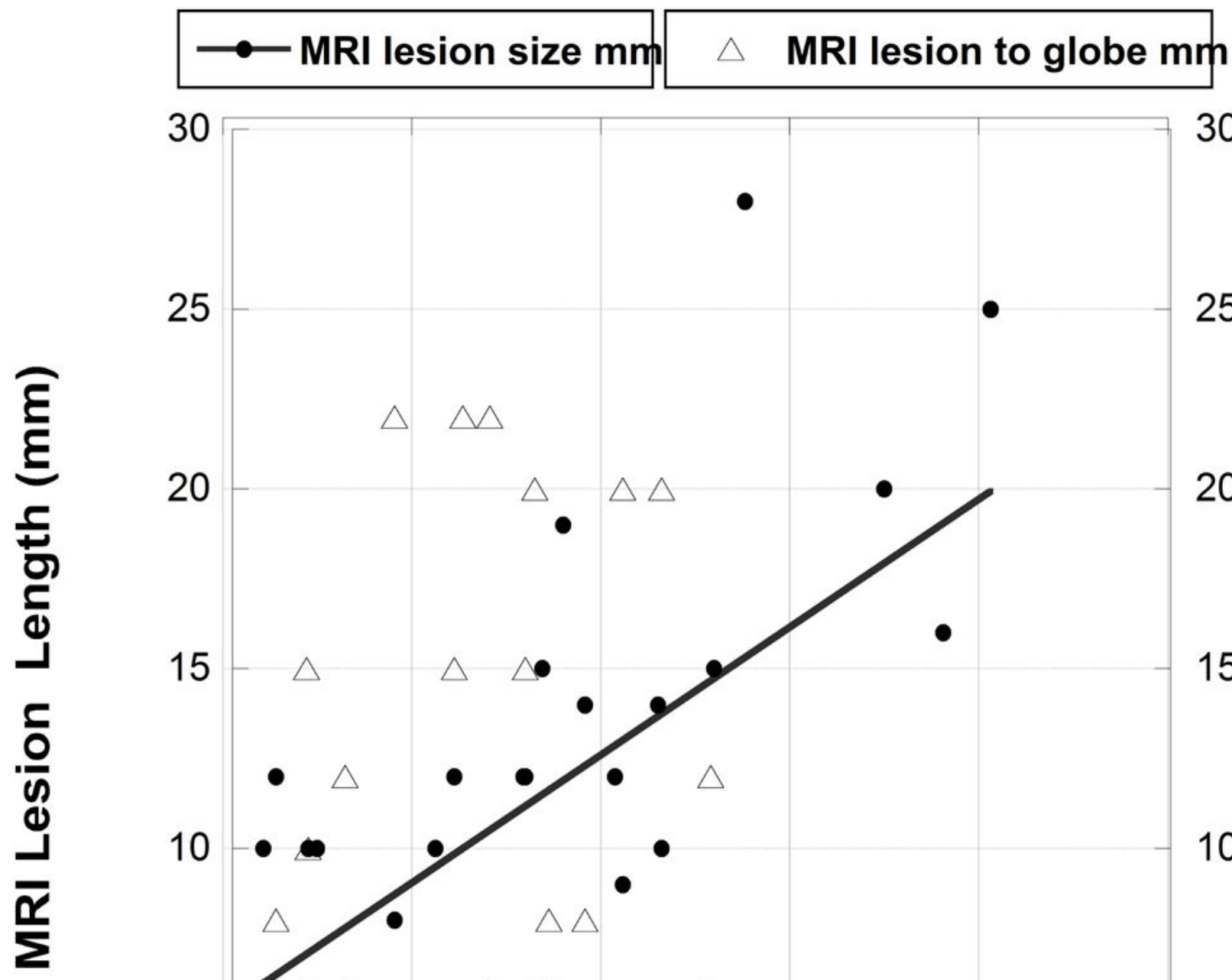

30
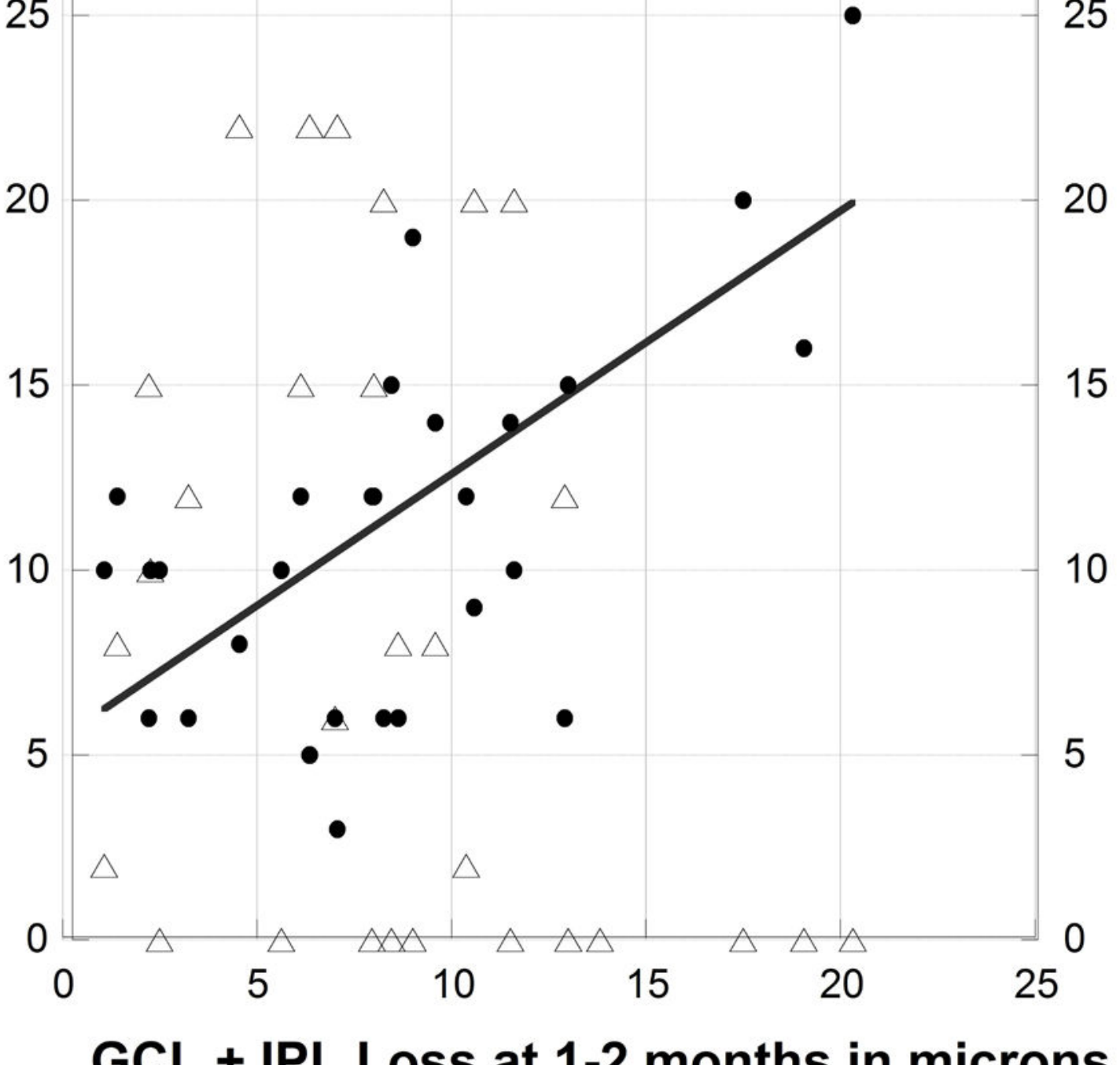

Figure 6.

Scatter plot of 3-D method derived GCL+IPL thinning (loss) at one-two months compared with length (solid circles) with linear fit line and distance from the globe (open triangles, and no fit line due to lack of correlation) of the MRI lesion of optic nerve. 


\section{Table 1}

Visual performance and GCL+IPL, RNFL thickness change over time

\begin{tabular}{|l|l|l|l|l|}
\hline & Presentation & Month 1-2 & Month 3 & $\begin{array}{l}\text { Month 6 } \\
\text { ( 5 months) }\end{array}$ \\
\hline LogMar visual acuity & $0.79 \pm 0.93$ & $0.11 \pm 0.27$ & $0.06 \pm 0.20$ & $0.02 \pm 0.10$ \\
\hline Visual field MD, dB & $-17.15 \pm 10.80$ & $-3.72 \pm 6.50$ & $-1.98 \pm 2.43$ & $-1.06 \pm 1.67$ \\
\hline Days from vision loss & $7 \pm 3.4$ & $38 \pm 13.3$ & $129 \pm 35.5$ & $304 \pm 128.2$ \\
\hline $\begin{array}{c}\text { GCL+IPL thickness, } \mu \mathrm{m} \\
\text { 3-D method }\end{array}$ & $82.4 \pm 8.8$ & $72.3 \pm 9.7$ & $72.5 \pm 12.0$ & $69 . \pm 11.6$ \\
\hline $\begin{array}{c}\text { GCL+IPL thickness, } \mu \mathrm{m} \\
\text { 2-D method }\end{array}$ & $81.2 \pm 14.1$ & $73.6 \pm 8.3$ & $72.0 \pm 12.0$ & $69.8 \pm 10.2$ \\
\hline $\begin{array}{c}\text { RNFL thickness, } \mu \mathrm{m} \\
\text { 2-D method }\end{array}$ & $114.4 \pm 43.4$ & $99.1 \pm 18.0$ & $87.0 \pm 11.7$ & $84.1 \pm 11.2$ \\
\hline $\begin{array}{c}\text { GCL+IPL thinning from prior visit, } \mu \mathrm{m} \\
\text { 3-D method }\end{array}$ & & $9.9 \pm 60^{*}$ & $3.0 \pm 3.0$ ** & $0.2 \pm 1.2$ *** \\
\hline
\end{tabular}

At presentation, unaffected fellow eyes had mean GCL+IPL for $2-\mathrm{D}$ method $=82.1 \pm 6.7 \mu \mathrm{m}$ and for 3 -D method $=82.2 \pm 7.0 \mu \mathrm{m}$, and $\mathrm{pRNFL}=$ $91.1 \pm 9.8 \mu \mathrm{m}$

One subject had the first follow up at six months

* Range $26.0 \mu \mathrm{m}$, Maximum 27.1, Minimum 1.1

***

Range $12.0 \mu \mathrm{m}$, Maximum 11.0, Minimum 0

$* * *$

Range $6.0 \mu \mathrm{m}$, Maximum 2.0, Minimum 0 